\title{
LEPROSY MIMICKING ANCA-ASSOCIATED VASCULITIS
}

Luciana Nunes Assis Daameche ${ }^{1, \star}$, Carlos Eduardo Lins ${ }^{1}$, Tayane Oliveira Pires ${ }^{1}$, Helga Moura Kehrle ${ }^{1}$, Dunya Bachour Basílio ${ }^{1}$, Gustavo de Paiva Costa ${ }^{1}$, Jamille Nascimento Carneiro ${ }^{1}$, Fernanda Freire Kosac ${ }^{1}$, Vanessa Burini Lobo ${ }^{1}$, Tiere Costa Bravim ${ }^{1}$, Isabela Taveira Mouzinho ${ }^{1}$, João Américo da Silveira ${ }^{1}$

1.Instituto Hospital de Base, Brasília (DF), Brazil.

*Corresponding author: lucianadaameche@hotmail.com

\section{BACKGROUND}

Diagnosis of primary vasculitis is challenging, since there are wide and heterogeneous clinical manifestations, with diagnostic criteria still scarce. Patients often present in limited forms of vasculitis, witch broadens the diagnostic possibilities. Patients with upper respiratory tract involvement often evolve with systemic disease. Most patients have nasal, sinus or ear involvement that may be present weeks or months before other symptoms. Peripheral neuropathy is also a common manifestation. Lepromatous and dimorphic forms of leprosy have similar clinical and serological characteristics and can simulate various rheumatic diseases. Leprosy is endemic in several regions of Brazil, and must be always in the differential diagnosis of atypical rheumatic diseases.

\section{CASE REPORT}

A 37-year-old female patient was admitted to the emergency department of otorhinolaryngology due to acute rhinosinusitis complicated by facial cellulitis. Intravenous antibiotic therapy was started. She reported recurrent rhinosinusitis and allergic symptoms since she was 17 years old. Three years ago, she developed ulnar mononeuritis and symmetric sensorimotor polyneuropathy of the lower limbs. Electromyography showed asymmetric motor sensory polyneuropathy, chronic axonal, with active denervation in distal muscles. She had a negative lymph smear for acid fast bacilli. During hospitalization, she developed arthritis of large and small joints, ulcerated and crusted lower limbs lesions (Fig. 1). She was treated with prednisone $60 \mathrm{mg}$, with marked improvement of her symptoms. Laboratory tests showed iron deficiency anemia, thrombocytosis, elevated erythrocyte sedimentation rate (ESR) and C-reactive protein (CRP), negative antinuclear antibody (ANA), antineutrophil cytoplasmic autoantibody (ANCA), and antiproteinase-3, normal renal function and urinary sediment. A thorax computed tomography (CT) was normal, and a sinuses CT showed pansinusitis (Fig. 2). In nasal videoendoscopy, crusted ulcerated lesions were seen. Skin and nasal ulcer biopsies were collected. Anatomopathological report of the nasal ulcer led to the diagnosis of Virchowian Hansen's disease (Fig. 3). She was referred to the Hansen's disease outpatient clinic at the Hospital Universitário de Brasilia for follow-up and treatment with rifampicin, clofazimine and dapsone. The patient authorized the use of images.

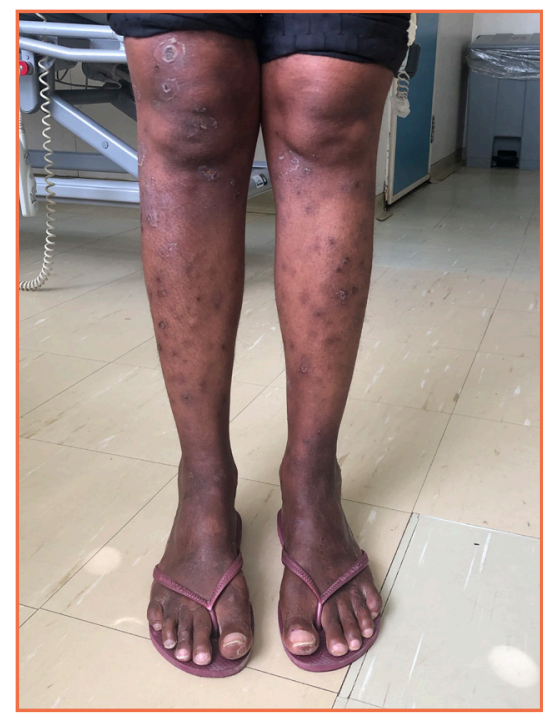

Figure 1. Papular lesions in the lower limbs, some ulcerated and with crusts. 


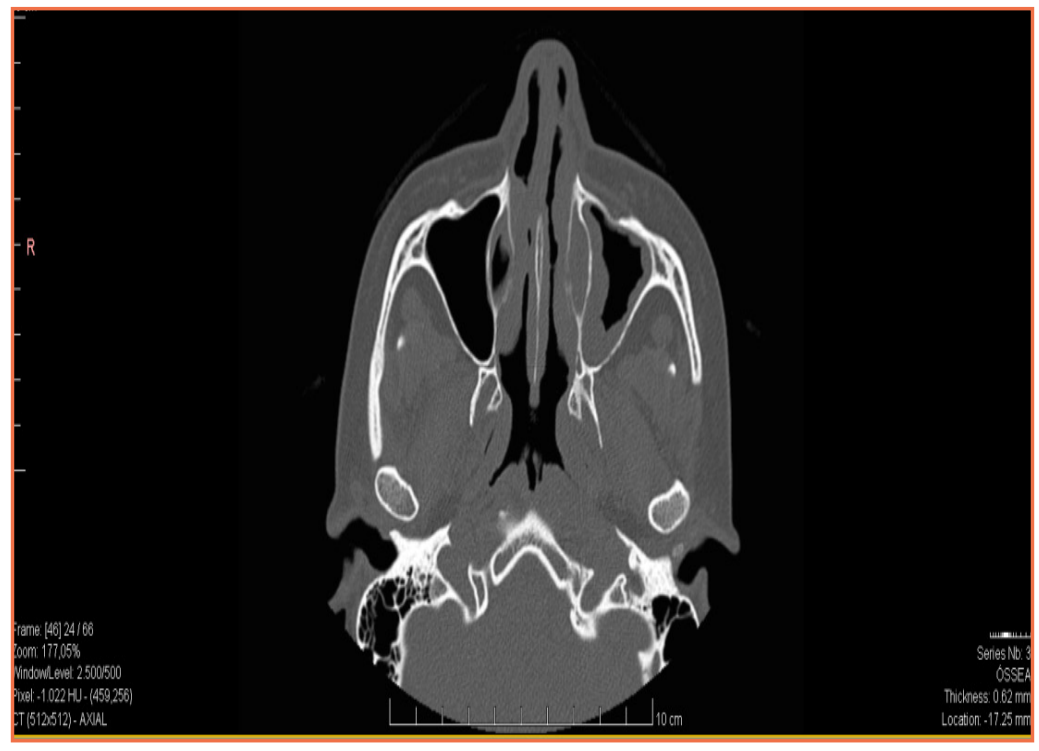

Figure 2. Sinus CT in axial section showing pansinusitis.

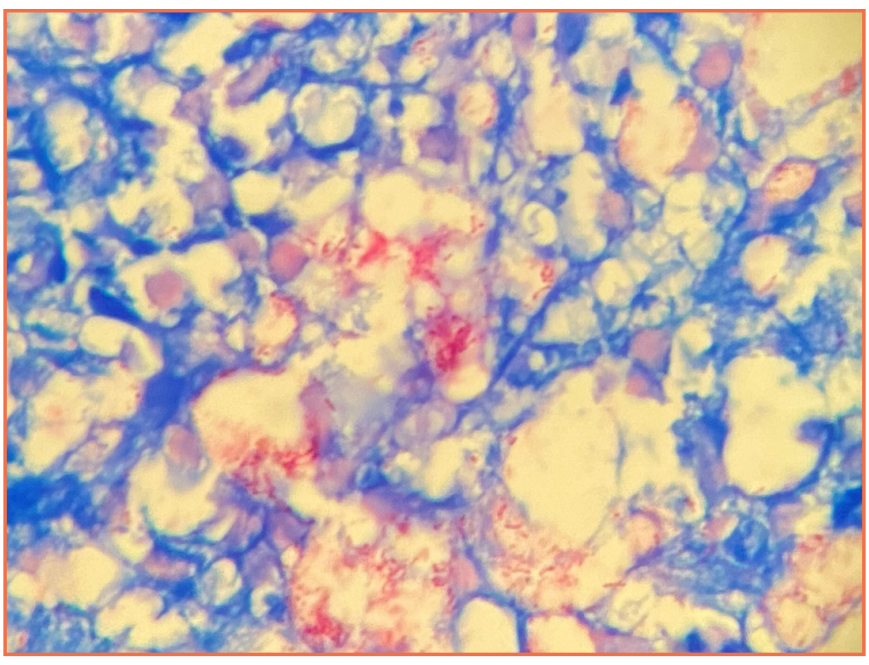

Figure 3. Fite-Faraco, $1000 \times$ - presence of numerous acid fast bacilli, forming globes.

\section{CONCLUSION}

Lepromatous and dimorphic forms can present with fever, a several of skin lesions, peripheral neuropathy, arthritis, chronic rhinosinusitis. Several of these manifestations improve on corticosteroids. Leprosy may simulate systemic vasculitis, and ANCA associated vasculitis. In endemic areas it is imperative to think in leprosy in patients with atypical forms of systemic rheumatic diseases. 